Ann. Génét. Sél. anim., I974, 6 (I), 29-34.

\title{
EFFETS DES GÈNES $s$ ET $k$ SUR LA CROISSANCE ET L'EFFICACITÉ ALIMENTAIRE CHEZ LE POUSSIN DANS 2 CROISEMENTS ET AVEC 2 NIVEAUX PROTÉIQUES
}

\author{
J. GUILLAUME \\ avec la collaboration technique de Liliane Derouer \\ Station de Recherches avicoles \\ Centre de Recherches de Tours, I. N.R. A., \\ Nouzilly, B. P. 1, 37380 Monnaie
}

\section{RÉSUMÉ}

Nous croisons des coqs hétérozygotes $(s k, S K)$ à des poules de génotype (sk-) appartenant soit à un croisement de type " ponte " (M), soit à un croisement de "reproductrices chair " $(\mathrm{J})$. Nous nourrissons les descendants femelles, élevés en cages individuelles, de régimes contenant soit 23,6 soit 15,5 p. roo de protéines brutes, et nous recherchons les effets des associations de gènes $s k$ et $S K$ sur la croissance et l'efficacité alimentaire dans les 2 contextes génétiques et les 2 contextes nutritionnels.

Outre les effets attendus de la carence en protéines et du croisement ( $\mathrm{M}$ ou J) sur le poids et l'indice de consommation, on observe des effets des gènes $s$ et $k$ sur le poids à Io j mais non sur l'indice de consommation : les poussins $s k$ pèsent 5,5 p. Ioo de plus que les poussins $S K$, quel que soit le régime, mais seulement dans le croisement J. Au-delà de to jours, seuls subsistent les effets du croisement et du régime alimentaire.

L'emploi des gènes $s$ et $k$ comme marqueurs du gène de nanisme $d w$ qui ralentit la croissance et diminue l'efficacité alimentaire est donc valable; il peut seulement biaiser les résultats sil'on s'intéresse au tout jeune poussin.

\section{I. - INTRODUCTION}

Le gène de nanisme récessif et lié au sexe dw (HUTT, I953) induit un retard de croissance progressif si bien qu'il est difficile de distinguer les phénotypes $d w$ et $D w$ avant 8 semaines. Pour étudier les effets de ce gène chez le jeune poussin, il faut donc avoir recours à des gènes marqueurs. Deux loci situés très près de $d w$ permettent cette distinction : le gène d'emplumement rapide $k$ et le gène induisant la présence de mélanines rousses dans les plumes $s$ (HUT'T, I959). Ils ont été utilisés à cette fin par plusieurs auteurs (Simon, I972, Touchburn et Blum, I972, Guil_aume, I973) qui n'ont d'ailleurs eu recours qu'aux associations $d w$ sk et $D w S K$.

Bien que l'on sache que les gènes $s$ et $k$ puissent avoir des effets propres sur les performances des animaux de o à 8 semaines (MéraT, I966) aucune étude n'a été entreprise pour voir si de tels effets existaient déjà chez le Poussin âgé de moins de 
8 semaines. C'est pourquoi nous avons cherché si le gène $k$ associé à $s$, dont l'effet était extériorisé ou non, pouvait modifier le développement pondéral et l'indice de consommation du poussin entre o et $20 \mathrm{j}$. Les poulets ont été nourris soit d'un aliment bien pourvu en protéines, soit d'un aliment carencé.

\section{II. - MATÉRIEL E'T MÉTHODES}

\section{Animaux}

L'expérience porte sur les poussins issus du croisement de coqs expérimentaux hétérozygotes pour les loci $s$ et $k(s k)$ accouplés à des poules d'origine commerciale de 2 types différents. Les coqs de phénotype $D w, C, i, K, S$ ont une formule variable pour les gènes contrôlant la forme de la crête, la couleur du tibia, etc. Il s'agit d'animaux de format moyen ( $3 \mathrm{~kg}$ environ). L'une des femelles utilisées est une pondeuse commerciale de type Rhode Island Red, la M 519 (SELAF, France) de formule $s k$; l'autre est la reproductrice de type " chair " nanifiée $J V 15$ (SELAF, France) ; elle est porteuse des mêmes gènes $s$ et $k$ mais la présence du gène $E$ empềche l'expression de $s$ dans la descendance.

Les poussins issus de ces 2 croisements (dénommés respectivement $M$ et $J$ pour les $M 519$ et les $J V 15$ ) sont sexés et classés en phénotypes $k$ et $K$ pour le croisement $\mathrm{J}$, sk et $S K$ pour le croisement $M$, les cas de recombinaison étant éliminés $4^{8}$ poulettes de chaque type sont mises en expérience.

\section{Aliments}

Les 2 régimes utilisés sont d'une part le régime 23 , bien pourvu en protéines, que nous avons employé antérieurement (GUILLAUME, 1972, 1973) et un régime isoénergétique, complet et équilibré sauf en ce qui concerne les protéines : le régime I $_{5}$ (tabl. I).

TABLEAU I

Composition des régimes utilisés

\begin{tabular}{|c|c|c|}
\hline & 15 & 23 \\
\hline Maïs $\ldots \ldots \ldots \ldots \ldots \ldots \ldots \ldots \ldots$ & 76,0 & 52,2 \\
\hline Tourteau de soja $50 \ldots \ldots \ldots \ldots \ldots$ & 16,3 & 36,5 \\
\hline Farine de hareng $70 \ldots \ldots \ldots \ldots$ & $0, \overline{5}$ & 1,0 \\
\hline DL-méthionine $\ldots \ldots \ldots \ldots \ldots \ldots$ & 0 & 0,35 \\
\hline Huile d'arachide $\ldots \ldots \ldots \ldots \ldots \ldots \ldots$ & 1 & 4 \\
\hline Phosphate bicalcique $\ldots \ldots \ldots \ldots \ldots \ldots$ & 2,5 & 2,5 \\
\hline Calcaire broyé $\ldots \ldots \ldots \ldots \ldots \ldots \ldots$ & 1,5 & 1,35 \\
\hline Sel iodé $\ldots \ldots \ldots \ldots \ldots \ldots \ldots \ldots \ldots$ & 0,5 & 0,5 \\
\hline Complément minéral et vitaminique ( $\left.{ }^{1}\right) \ldots$. & 1,7 & 1,7 \\
\hline Analyse & & \\
\hline Énergie métabolisable $\mathrm{kcal} / \mathrm{g} \ldots \ldots \ldots \ldots$ & 3,08 & 3,08 \\
\hline$N \times 6,25 \ldots \ldots \ldots \ldots \ldots \ldots \ldots \ldots \ldots$ & 15,5 & 23,6 \\
\hline 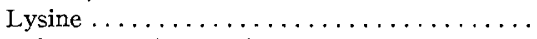 & 0,75 & 1,41 \\
\hline Acides aminés soufrés $\ldots \ldots \ldots \ldots \ldots$ & 0,57 & 1,13 \\
\hline
\end{tabular}

(1) Apportant pour $100 \mathrm{~kg}$ : Vitamine A: $800000 \mathrm{UI}$, Vitamine $\mathrm{D}_{3}$ : 100000 , vitamine $E 0,25 \mathrm{~g}$, vitamine $\mathrm{K}_{3}: 0,2 \mathrm{~g}$, riboflavine $: 1 \mathrm{~g}$, nicotinamide: $2 \mathrm{~g}$, pantothénate de calcium : $0,8 \mathrm{~g}$, acide folique : $0,15 \mathrm{~g}$, vitamine $\mathrm{B}_{12}$ : $1 \mathrm{mg}$, BHT : $10 \mathrm{~g}$, avoine broyée q.s.p : $1000 \mathrm{~g}$, cuivre: $0,28 \mathrm{~g}$, fer : $28 \mathrm{~g}$, iode: $0,14 \mathrm{~g}$, manganèse : $8,66 \mathrm{~g}$, zinc : $7,92 \mathrm{~g}$ et cobalt : $0,027 \mathrm{~g}$. 


\section{Élevage}

Comme dans les essais antérieurs, (Guillaume, 1973) nous élevons toutes les poulettes dans des cages individuelles de la naissance à l'âge de $20 \mathrm{j}$. Ces cages sont placées dans un bâtiment conditionné où le chauffage à air chaud maintient une température de $32^{\circ} \pm \mathrm{I}$ et où $\mathrm{l}^{\prime}$ hygrométrie est de $70 \mathrm{p}$. I00 \pm 5 . La température ambiante est abaissée de $2^{\circ} \mathrm{C}$ par semaine.

L'analyse des résultats porte sur le poids et l'indice de consommation individuels à Io et $20 \mathrm{j}$. Par une analyse de variance du dispositif factoriel $2^{3}$ on cherche à mettre en évidence les effets du croisement ( $M$ ou J) de l'aliment (I 5 ou 23) et du phénotype "emplumement rapide " ( $s k$ ou $S K$ ) ainsi que de leurs interactions.

\section{III. - RÉSULTATS ET DISCUSSION}

L'ensemble des résultats figure au tableau 2. Les faits les plus nets qui s'en dégagent sont les effets du croisement et de l'aliment : tous deux influencent très nettement aussi bien la croissance que l'indice de consommation $(p<0,00 I)$. En revanche, le phénotype " emplumement " (marqueur) n'a pas d'effet global au niveau de ces 2 variables.

TABLEAU 2

Principaux résultats

\begin{tabular}{|c|c|c|c|c|c|c|}
\hline \multirow{2}{*}{ Age } & \multirow{2}{*}{ Croisement } & Protéines \% Régime & \multicolumn{2}{|c|}{15,5} & \multicolumn{2}{|c|}{23,6} \\
\hline & & Gènes marqueurs & $S K$ & $s k$ & $S K$ & $s k$ \\
\hline \multirow{2}{*}{$\begin{array}{c}0 \text { à } 10 \\
\text { jours }\end{array}$} & $\mathbf{J}$ & $\begin{array}{l}\text { Consommation }(g / j) \ldots \ldots \\
\text { Gain de poids }(g / j) \ldots \ldots \\
\text { Indice de Consnmmation }\end{array}$ & $\begin{array}{c}20,1 \\
8,2 \pm 0,2 \\
2,48 \pm 0,05\end{array}$ & $\begin{array}{c}20,8 \\
8,7 \pm 0,2 \\
2,39 \pm 0,02\end{array}$ & $\begin{array}{c}18,7 \\
10,2 \pm 0,3 \\
1,84 \pm 0,02\end{array}$ & $\begin{array}{c}19,0 \\
10,6 \pm 0,3 \\
1,80 \pm 0,02\end{array}$ \\
\hline & M & $\begin{array}{l}\text { Consommation }(g / j) \ldots \\
\text { Gain de poids }(g / j) \ldots \ldots \\
\text { Indice de consommation }\end{array}$ & $\begin{array}{c}17,9 \\
6,7 \pm 0,2 \\
2,70 \pm 0,04\end{array}$ & $\begin{array}{c}17,8 \\
6,7 \pm 0,2 \\
2,67 \pm 0,04\end{array}$ & $\begin{array}{c}16,7 \\
8,9 \pm 0,2 \\
1,91 \pm 0,03\end{array}$ & $\begin{array}{c}16,3 \\
8,4 \pm 0,2 \\
1,95 \pm 0,03\end{array}$ \\
\hline \multirow{2}{*}{$\begin{array}{c}10 \text { à } 20 \\
\text { jours }\end{array}$} & $\mathrm{J}$ & $\begin{array}{l}\text { Consommation }(g / j) \ldots \ldots \\
\text { Gain de poids }(g / j) \ldots \ldots \\
\text { Indice de consommation }\end{array}$ & $\begin{array}{c}38,9 \\
15,5 \pm 0,3 \\
2,53 \pm 0,03\end{array}$ & $\begin{array}{c}39,0 \\
15,6 \pm 0,2 \\
2,50 \pm 0,03\end{array}$ & $\begin{array}{l}39,9 \\
19,8 \pm 0,4 \\
2,03 \pm 0,04\end{array}$ & $\begin{array}{c}39,5 \\
20,1 \pm 0,02 \\
1,97 \pm 0,02\end{array}$ \\
\hline & M & $\begin{array}{l}\text { Consommation }(g / j) \ldots \ldots \\
\text { Gain de poids }(g / j) \ldots \ldots \\
\text { Indice de consommation }\end{array}$ & $\begin{array}{c}32,8 \\
12,2 \pm 0,3 \\
2,70 \pm 0,04\end{array}$ & $\begin{array}{c}32,5 \\
11,6 \pm 0,2 \\
2,81 \pm 0,04\end{array}$ & $\begin{array}{c}33,9 \\
16,0 \pm 0,3 \\
2,11 \pm 0,03\end{array}$ & $\begin{array}{c}32,5 \\
15,7 \pm 0,3 \\
2,08 \pm 0,04\end{array}$ \\
\hline
\end{tabular}

De ro à 20 jours, les gènes $s$ et $k$ n'ont aucune influence. De o à ro jours toutefois on observe deux interactions significatives; pour le gain de poids d'abord, il existe une interaction croisement $\times$ marqueur; les poulettes $s k$ ont la même croissance que les sujets $S K$ dans le croisement M, tandis que dans le croisement $\mathrm{J}$ les poulettes $k$ ont un poids plus élevé que les poulettes $K(p<0,05)$. Cet effet favorable de $k$ se retrouve aussi au niveau de l'efficacité alimentaire mais de façon moins nette. 
Il existe également une interaction croisement $x$ aliment $(p<0,0$ I $)$. La carence en protéines élève l'indice de consommation de façon beaucoup plus accusée dans le croisement M que dans le croisement J. Il faut préciser ici les effets de la carence en protéines sur la consommation : loin de déprimer l'ingéré comme on l'observe dans les expériences à long terme, elle l'augmente durant la période initiale (o à Io j). Cette surconsommation, déjà connue, (GuIllaume, FEndRy et IMBACh, I965) est fonction du génotype: elle est surtout nette chez les poulettes à croissance lente du croisement $M$. Elle ne se traduit pas par une diminution de l'écart de poids vis-à-vis des oiseaux nourris de régime 23 , mais par une détérioration plus accentuée de l'indice de consommation.

L'association dont nous recherchions un effet éventuel sur la croissance et l'efficacité alimentaire est donc bien susceptible de modifier modestement les performances du jeune Poussin; le résultat va dans le sens de celui de MÉRAT, I962 qui observait lui aussi un poids plus élevé des poulettes à emplumement rapide, en utilisant, il est vrai, des animaux à croissance très lente et beaucoup plus âgés. Il paraît donc plausible que le léger gain $(5,3 \mathrm{~g})$ trouvé par MÉRAT à 8 semaines ne soit autre que la différence de $5 \mathrm{~g}$ que nous détectons à l'âge de ro jours (où il représente $5 \mathrm{p}$. Ioo du poids gagné depuis la naissance) et qui ne s'accentue pas de ro à 20 jours. Nos données permettent en tous cas de compléter et de nuancer ce résultat : "l'avance à Io jours " est indépendante de l'aliment; elle n'est toutefois présente que dans un croisement, ce qui rend peu probable l'hypothèse d'un effet pléiotropique direct par le biais de la thermorégulation.

L'hypothèse d'un linkage de $k$ ou de $s$ avec des gènes agissant sur la croissance ou l'appétit est plus vraisemblable.

Le marquage de $d w$ par $s$ et $k$ ne peut toutefois guère modifier les effets du premier gène : la différence de $5 \mathrm{~g}$ que nous avons mise en évidence grâce à un dispositif qui s'est avéré très précis, est en effet très mince comparée aux différences de poids qu'induit ce gène de nanisme. En deçà de ro jours seulement, on risque, pour certains critères tels que le gai n de poids, de fausser la validité-des tests d'hypothèses : l'effet propre des gènes marqueurs pourrait entraîner une surestimation du risque de premier ordre. Il conviendrait alors de marquer le gène $d w$ sur une partie des animaux par $s k$ et une autre par $S K$.

Reçu pour publication en novembe 1973.

\section{SUMMARY}

\section{EFFECTS OF $S$ AND $k$ GENES ON GROWTH AND FEEDING EFFICIENCY OF CHICKEN IN TWO CROSSES AND WITH TWO PROTEIN LEVELS}

Heterozygous $(s k / S K)$ cocks were mated to hens of the (sk) genotype from either a laying type cross (M) or a broiler female cross $(\mathrm{J})$. The female progeny, reared in individual cages, were fed with rations containing either 23.6 or 15.5 p. roo raw protein. The effects of the associated genes $s k$ and $S K$ on growth and feed efficiency were looked for in the 2 genetic and nutritional backgrounds.

Besides the expected effects of protein shortage and of the cross ( $\mathrm{M}$ or $\mathrm{J}$ ) on weight and teed efficiency, an effect of the $s$ and $k$ genes is observed on weight at Io days but no on efficiency : $s k$ chicks weigh 5.5 p. Ioo more than $S K$ ones, irrespective of the ration, but only in the J cross. After ro days of age, only the effects of the cross and ration remain. 
The use of the $s$ and $k$ genes as markers for the dwarf gene dw wich depresses growth and lowers feed efficiency is therefore valuable; it can cause a bias only on results concerning very young chicks.

\section{RÉFÉRENCES BIBLIOGRAPHIQUES}

Guillaume J., 1973. Particularités physiologiques et nutritionnelles du Poussin nain dw. IVe Europ. Poult. Conf. London, 557-562.

Guillaume J., Fendry M., IмвасH B., 1965. Le rôle des protides dans l'utilisation des aliments du Poussin. II. Influence du mode de distribution des aliments sur la consommation d'énergie. Ann. Biol. anim. Bioch Biophys., 5, 293-308.

Hutt F. B., 1953. Sex-linked dwarfism in the fowl. Genetics, 38, 670.

HutT F.-B., 1959. Sex-linked dwarfism in the fow. J. Heredity, 50, 209-22I.

MÉrAT P., I962. Quelques relations entre caractères extérieurs à hérédité simple et productivité. XII World's Poult. Cong., Sydney, $7 \mathrm{I}-76$.

Simon J., r972. Influence du gène de nanisme (dw), du cou nu $(\mathrm{Na})$ et du rythme d'alimentation sur la croissance et le comportement alimentaire đu Poulet. Ann. Génét. Sél. anim., 4, 305-310.

Touchburn S.-P., Blum J.-C., 1972. Effects of the genes for dwarfism (dw) and naked neck (Na) on chick growth and lipid metabolism. Ann. Génét. Sél. anim., 4, 3II-315. 